\title{
The nature of the coronae of accreting black holes
}

\section{Anne Lohfink ${ }^{* \dagger}$}

Institute of Astronomy, University of Cambridge

E-mail: alohfink@ast.cam.ac.uk

Accreting black holes are powerful sources of radiation. The conversion of gravitational energy into radiation is thought to take place in an accretion disk-corona system just outside the black hole. In this system, thermal accretion disk photons are upscattered in a corona of hot electrons situated above the accretion disk, producing X-rays. The nature of this Comptonizing corona remains a key open question in astrophysics. I will discuss recent INTEGRAL and NuSTAR results on galactic black hole binary and AGN coronae.

11th INTEGRAL Conference Gamma-Ray Astrophysics in Multi-Wavelength Perspective 10-14 October 2016

Amsterdam, The Netherlands

* Speaker.

${ }^{\dagger}$ A footnote may follow. 


\section{Introduction to Black Hole Coronae}

The corona is a region of hot electrons in the immediate vicinity of the black hole where the $\mathrm{X}$ ray continuum is thought to be produced. X-rays are created via the inverse-Compton upscattering of thermal accretion disk emission. The energy distribution of the electrons is usually assumed to be thermal, but indications for a non-thermal contribution have been clearly detected in X-ray binaries (e.g., [1]). Despite the basic picture being well established, much about the corona is not yet understood. Among the many unknowns is its geometry. Several geometries have been suggested, including a slab geometry where the corona sandwiches the disk, a spherical layout where the corona surrounds the black hole and a lamp-post geometry, where the corona is pointlike above the black-hole-disk system (Fig.1). Aside from the geometry, the corona is defined by the balance of heating and cooling. The heating is thought to take place magnetically, while the corona cools mostly via radiation.

In recent years, our knowledge of the corona has been improving. For example, we now know the coronal sizes and temperatures better than ever. This knowledge is allowing us to confront ideas such as the regulation of the coronal temperature via electron-positron pairs with much better data. Here we discuss in more detail the progress that has been made and what can be learnt from it. The focus is on active galactic nuclei (AGN), as the state transitions in black hole X-ray binaries make the interpretation of the data more difficult.

A)

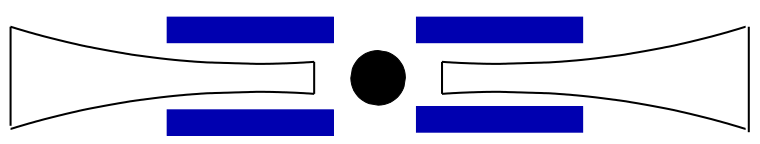

B)

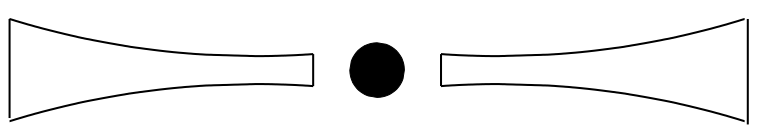

C)

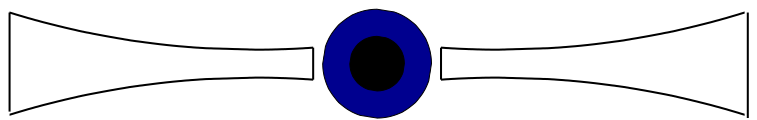

Figure 1: Different possible coronal geometries: A) Slab, B) Lamp-post, C) Spherical. The corona is shown in blue, together with the central black hole and the accretion disk.

\section{The knowns}

\subsection{Measurements of coronal sizes}

Several techniques have been employed to constrain the size of the X-ray corona. The first two methods use the fact that part of the $\mathrm{X}$-ray continuum emission is reprocessed by the accretion 
disk in a process called X-ray reflection [2]. This produces characteristic atomic spectral features in the X-ray spectrum. The features of atomic origin are modified by the relativistic effects taking place in the vicinity of the black hole. Comparing the resulting spectra with simulated template spectra allows us to constrain the geometry, and thereby the size, of the innermost region. For example, work by [3] shows that the corona in Mrk 335 must be smaller than $3 R_{\mathrm{g}}$. Another quantity associated with $\mathrm{X}$-ray reflection is the time delay between changes in the primary continuum and the response of the reprocessed emission. X-ray reverberation measurements that study this delay can be used to place limits on the size of the coronal region as well. [4] studied X-ray reverberation in a sample of radio-quiet $\mathrm{AGN}$ and found that the inferred coronal sizes are $\leqslant 10 R_{\mathrm{g}}$.

Another way the size of the corona can be measured is by investigating the spectral energy distributions of AGN. This method makes use of the broad-band nature of Comptonization. The $\mathrm{X}$-ray spectra are fitted with a spectral Comptonization model that contains the seed photons such as compps [5] or eqpair [6]. This best-fit model is then extrapolated into the optical/UV region and compared to the measurements there. [7] and [8] found that this comparison reveals a massive excess in the measured optical/UV emission relative to what is predicted by the X-ray Comptonization model, i.e. the seed photons alone. This discrepancy can be readily explained by a very spatially compact corona where only a small fraction of the total accretion disk emission is Compton upscattered.

The final method makes minimal assumptions: in X-ray microlensing the size of the emission region is estimated from the amplitude of the variability in the lensed images of a quasar. Those studies also indicate a primary X-ray emission region size of $\sim 10 R_{\mathrm{g}}[9,10]$.

Despite the different assumptions made by the various techniques, they all point towards a very compact corona $\left(R<10 R_{\mathrm{g}}\right)$. Combined with the high radiative output of this region, this compactness has implications for the physical processes taking place there, as we will discuss in Section 3.

\subsection{Coronal temperatures}

The X-ray spectrum not only constrains the size of the corona but also allows us to measure the high-energy turnover, i.e. the temperature of the corona. With NUSTAR, INTEGRAL and SwiftBAT currently operating, the number of temperature measurements is growing. Figure 2 shows the temperature distributions from the three samples. The top panel shows the distribution for a sample where the temperature measurements stem from joint INTEGRAL and Swift modeling [11]. In the middle panel only INTEGRAL was used to make the measurements [12] and finally the bottom panel shows all those temperatures derived from NUSTAR data ([13] with additions described in the next Section). All three samples indicate a non-uniform distribution with a peak at around $\sim 50 \mathrm{keV}$, a tail towards higher temperatures and a sharp drop-off at lower temperatures. Despite those common characteristics of the distributions, there are some subtle differences. These could be caused by the different energy ranges of the data used, or by the different sample selections; the first two samples are hard X-ray selected while the NUSTAR sample mostly consists of objects with large amounts of X-ray reflection.

In addition to determining the average temperature, both the INTEGRAL [14] and NUSTAR [15] studies found the temperature to be time-variable. 

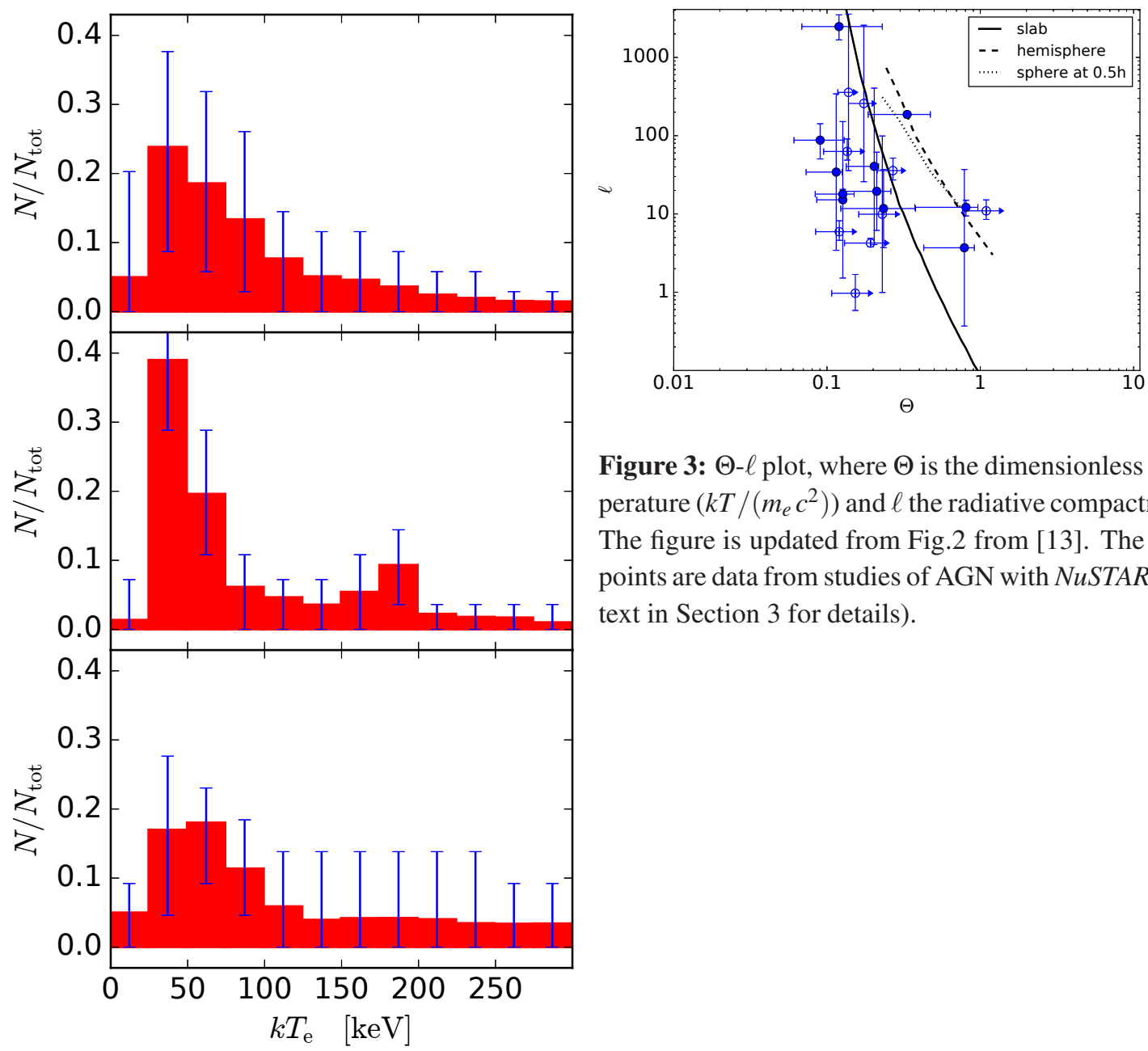

Figure 3: $\Theta-\ell$ plot, where $\Theta$ is the dimensionless temperature $\left(k T /\left(m_{e} c^{2}\right)\right)$ and $\ell$ the radiative compactness. The figure is updated from Fig.2 from [13]. The blue points are data from studies of AGN with NuSTAR (see text in Section 3 for details).

Figure 2: Distribution of electron temperatures from several samples: Top Panel: INTEGRAL+Swift sample of 37 sources [11], Middle Panel: INTEGRAL sample of 28 sources [12], Bottom Panel: NUSTAR sample of 24 sources [13]. All three distributions show a clear peak at approximately $50 \mathrm{keV}$ and very few sources below $25 \mathrm{keV}$ and above $150 \mathrm{keV}$.

\section{The potential importance of pairs}

The rapidly growing number and quality of coronal temperature measurements and the shape of the distribution that is emerging raises the question as to what sets the temperature of the corona.

One possibility that has been discussed $[16,17,18,19,6,20]$ is that the radiative compactness of the corona, $\ell=L / R \times \sigma_{\mathrm{T}} /\left(m_{e} c^{3}\right)$, plays an important role. Once the temperature rises above a certain value for a given compactness, the corona will not heat up any further. Instead, large numbers of $e^{+} / e^{-}$pairs will be created, which cool the corona back down, effectively provid- 
ing an upper limit in the temperature-compactness plane. The limiting temperature as a function of compactness depends on the coronal geometry (Fig. 3). [13] placed the NUSTAR temperature measurements in the temperature-compactness plane, using the best mass and coronal size measurements available, and found that the values are consistent with pairs limiting the coronal temperatures, i.e. no values where found to the right of the line. This held even when accounting for the gravitational redshifting the temperature measurements are subject to. Here (Fig. 3), we show an updated version of Fig. 2 from [13] including additional data from PG 1211+143 [21], Cen A [22], PG 1247+367 [23], 3C 273 [24], and 4C 74.26 (Lohfink et al., in prep). These data points were obtained in a similar fashion to those already presented in [13]. Even when including these additional measurements, it is still apparent that all data are in agreement with an upper limit for the temperature set by pairs. It is also clear that not all sources have the maximum theoretically allowed temperature, which could be explained by a small non-thermal contribution to the electron distribution. This higher energy tail could lead to run-away pair production at lower temperatures pushing the curves in Fig. 3 to lower temperatures.

\section{Summary}

We conclude that, even when additional data is added, our results remain consistent with pair creation acting as a thermostat on the corona. It is also likely that non-thermal electrons are potentially important in determining the coronal temperature, as indicated by those sources that have temperatures well below the thermal pair limit boundary. Work investigating this in greater detail is ongoing, alongside studies exploring the nature of the subtle differences in the electron temperature distributions.

\section{Acknowledgments}

AL acknowledges support from ERC Advanced Grant FEEDBACK 340442.

\section{References}

[1] M. C. Bel, P. Sizun, A. Goldwurm, J. Rodriguez, P. Laurent, A. A. Zdziarski et al., The broad-band spectrum of Cygnus X-1 measured by INTEGRAL, Astronomy and Astrophysics 446 (sep, 2005) 591-602, [0 509851$]$.

[2] I. M. George and A. C. Fabian, X-ray reflection from cold matter in Active Galactic Nuclei and X-ray binaries, Monthly Notices of the Royal Astronomical Society 249 (mar, 1991) 352-367.

[3] M. L. Parker, D. R. Wilkins, A. C. Fabian, D. Grupe, T. Dauser, G. Matt et al., The NuSTAR spectrum of Mrk 335: extreme relativistic effects within two gravitational radii of the event horizon?, Monthly Notices of the Royal Astronomical Society 443 (jul, 2014) 1723-1732.

[4] E. Kara, W. N. Alston, A. C. Fabian, E. M. Cackett, P. Uttley, C. S. Reynolds et al., A global look at $X$-ray time lags in Seyfert Galaxies, Monthly Notices of the Royal Astronomical Society 462 (may, 2016) 511-531, [1605.02631].

[5] J. Poutanen, K. N. Nagendra and R. Svensson, Green's matrix for Compton reflection of polarized radiation from cold matter, Monthly Notices of the Royal Astronomical Society 283 (1996) 892-904. 
[6] P. S. Coppi, The Physics of Hybrid Thermal/Non-Thermal Plasmas, High Energy Processes in Accreting Black Holes (1999).

[7] D. R. Ballantyne, J. M. Bollenbacher, L. W. Brenneman, K. K. Madsen, M. Baloković, S. E. Boggs et al., NUSTAR reveals the Comptonizing corona of the broad-line radio galaxy $3 C$ 382, The Astrophysical Journal 794 (sep, 2014) 62.

[8] A. Lohfink, P. Ogle, F. Tombesi, D. Walton, M. Balokovic, A. Zoghbi et al., The Corona of the Broad-Line Radio Galaxy 3C 390.3, The Astrophysical Journal 814 (oct, 2015) 9, [1510. 01333 ].

[9] C. W. Morgan, L. J. Hainline, B. Chen, M. Tewes, C. S. Kochanek, X. Dai et al., Further evidence that quasar X-ray emitting regions are compact: X-ray and optical microlensing in the lensed quasar Q J0158-4325, The Astrophysical Journal 756 (sep, 2012) 52.

[10] C. L. MacLeod, C. Morgan, A. Mosquera, C. Kochanek, M. Tewes, F. Courbin et al., A Consistent Picture Emerges: A Compact X-ray Continuum Emission Region in the Gravitationally Lensed Quasar SDSS J0924+0219, eprint arXiv:1501.07533 (2015).

[11] A. Malizia, M. Molina, L. Bassani, J. B. Stephen, A. Bazzano, P. Ubertini et al., The INTEGRAL high energy cut-off distribution of type 1 AGN, 1401.3647.

[12] P. Lubiński, V. Beckmann, L. Gibaud, S. Paltani, I. E. Papadakis, C. Ricci et al., A comprehensive analysis of the hard X-ray spectra of bright Seyfert galaxies, Monthly Notices of the Royal Astronomical Society 458 (feb, 2016) 2454-2475, [1602 . 08402 ].

[13] A. C. Fabian, A. Lohfink, E. Kara, M. L. Parker, R. Vasudevan and C. S. Reynolds, Properties of AGN coronae in the NUSTAR era, Monthly Notices of the Royal Astronomical Society 451 (may, 2015) 4375-4383, [1505.07603].

[14] P. Lubinski, A. A. Zdziarski, R. Walter, S. Paltani, V. Beckmann, S. Soldi et al., Extreme flux states of NGC 4151 observed with INTEGRAL, Monthly Notices of the Royal Astronomical Society 408 (may, 2010) 1851-1865, [1005.0842].

[15] F. Ursini, P. O. Petrucci, G. Matt, S. Bianchi, M. Cappi, B. De Marco et al., High-energy monitoring of NGC 4593 with XMM-Newton and NuSTAR. X-ray spectral analysis, Monthly Notices of the Royal Astronomical Society 463 (aug, 2016) 382-392, [1608 . 02847].

[16] R. Svensson, Steady mildly relativistic thermal plasmas - Processes and properties, Monthly Notices of the Royal Astronomical Society 209 (1984) 175-208.

[17] A. A. Zdziarski and A. P. Lightman, Nonthermal electron-positron pair production and the 'universal' X-ray spectrum of active galactic nuclei, The Astrophysical Journal 294 (jul, 1985) L79.

[18] P. Pietrini and J. H. Krolik, The Inverse Compton Thermostat in Hot Plasmas near Accreting Black Holes, The Astrophysical Journal 447 (jul, 1995) 526.

[19] B. E. Stern, J. Poutanen, R. Svensson, M. Sikora and M. C. Begelman, On the Geometry of the X-Ray emitting Region in Seyfert Galaxies, The Astrophysical Journal 449 (aug, 1995) 13.

[20] J. B. Dove, J. Wilms, M. Maisack and M. C. Begelman, Self-consistent Thermal Accretion Disk Corona Models for Compact Objects. II. Application to Cygnus X-1, The Astrophysical Journal 487 (oct, 1997) 759-768.

[21] A. Zoghbi, J. M. Miller, D. J. Walton, F. A. Harrison, A. C. Fabian, C. S. Reynolds et al., NuSTAR Reveals Relativistic Reflection But No Ultra-Fast Outflow In The Quasar PG 1211+143, The Astrophysical Journal Letters 799 (jan, 2015) 5, [1501.01663]. 
[22] F. Fuerst, C. Mueller, K. K. Madsen and L. Lanz, NuSTAR and XMM-Newton Observations of the Hard X-Ray Spectrum of Centaurus A, The Astrophysical Journal 819 (nov, 2015) 13, [1511.01915].

[23] G. Lanzuisi, M. Perna, A. Comastri, M. Cappi, M. Dadina, A. Marinucci et al., NuSTAR reveals the extreme properties of the super-Eddington accreting SMBH in PG 1247+267, Astronomy \& Astrophysics $\mathbf{5 9 0}$ (apr, 2016) 12, [1604 .02462].

[24] K. K. Madsen, F. Fuerst, D. J. Walton, F. A. Harrison, K. Nalewajko, D. R. Ballantyne et al., $3 C 273$ with NuSTAR : Unveiling the active galactic nucleus, The Astrophysical Journal 812 (oct, 2015) 14. 